\title{
DISPOSITIVO AUTOMATIZADO DE REMOÇÃO DE CASCÃO NAS LANÇAS DOS CONVERTEDORES LD RCL - MVL -01 - INPI: № 8603033-7*
}

Emilio Acciarito Filho ${ }^{1}$ Aristides Alves Barbosa ${ }^{2}$

\section{Resumo}

O presente dispositivo, tem como objetivo promover de modo contínuo e automatizado a remoção do cascão incandescente aderido nas superfícies das lanças de injeção de oxigênio e gases inertes utilizadas nos processos dos convertedores LD. Constituído de um conjunto mecânico de ferramentas de remoção, posicionadas em um suporte articulado bi partido e simétrico, acionados por cilindros pneumáticos e interligados ao sistema computorizado (PLC) do carro de movimentação vertical das lanças via sensores indutivos de posição; Resultando na maximização das utilizações dessas lanças e consequente aumento da produtividade, com redução de custo e maior segurança operacional nos processos LD.

Palavras-chave: Dispositivo de remoção; Cascão de lanças; Processos dos convertedores LD; Segurança; Produtividade.

\section{SKULL REMOTION DEVICE IN LANCES OF BOF PROCESS - SRD - MVL - 01 \\ Abstract}

This device, has as objective to promote continuously (every ascent of lances) removing the adhered incandescent skull on the surfaces of the injection lances oxygen and inert gases in BOF process, employing a set of mechanical removal tools, positioned in an symmetric support and bi party, driven by pneumatic cylinders and linked to the computerized system (PLC) car vertical movement of lances; Resulting in maximizing uses of lances and consequent increase in productivity, cost reduction and greater operational safety in BOF process.

Keywords: Remotion device; Lances skull; BOF process; Safety; Productivity.

1 Engenheiro Ind. Metalúrgico, M.M.E., Gerente Técnico comercial, Metalúrgica Vulcano Ltda., Barra Mansa, RJ, Brasil.

2 Técnico industrial, especialista em manutenção mecânica, Metalúrgica Vulcano Ltda, Barra Mansa, RJ, Brasil. 


\section{INTRODUÇÃO}

A formação do cascão (mistura de escória e aço) e sua aderência na superfície das lanças de injeção de oxigênio e gases inertes, utilizadas nos processos de refino e "slag splash" respectivamente nos processos dos convertedores LD, é inerente as condições desses processos, tendo sua maior ou menor incidência em função de alguns aspectos como, composição química do gusa líquido (principalmente a variação do teor de silício), adições de sucata (qualidade), viscosidade da escória, altura das lanças em relação ao banho, pressão / vazão do gás injetado, desgaste dos furos da "cabeça" das lanças e outros (1), (2) e (3). A deposição deste cascão de modo acumulativo na superfície das lanças, aumenta o seu diâmetro e peso (fig.1).

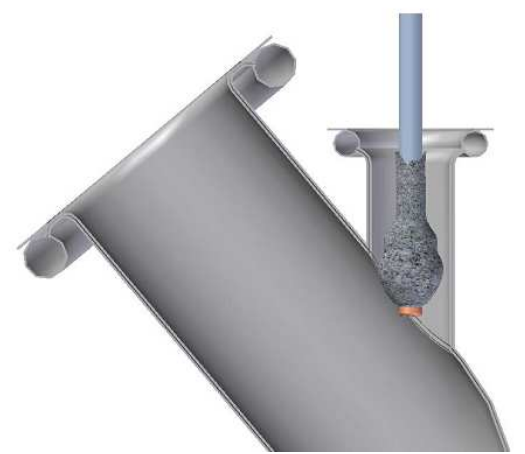

Figura 1 - llustração de um cascão típico aderido em lança convencional (1)

Com esta deposição de cascão, aumenta as dificuldades operacionais de movimentação dessas lanças nas operações de introdução e retirada do interior dos convertedores em suas passagens pelo duto denominado de "camisa da lança"; Acarretando alta frequência de trocas (lanças) com efeitos indesejáveis como perda de produtividade, aumento de custos e insegurança operacional, pela necessidade de limpezas / remoção desse cascão que é usualmente realizado de modo manual (via oxi - corte) fig. 2.

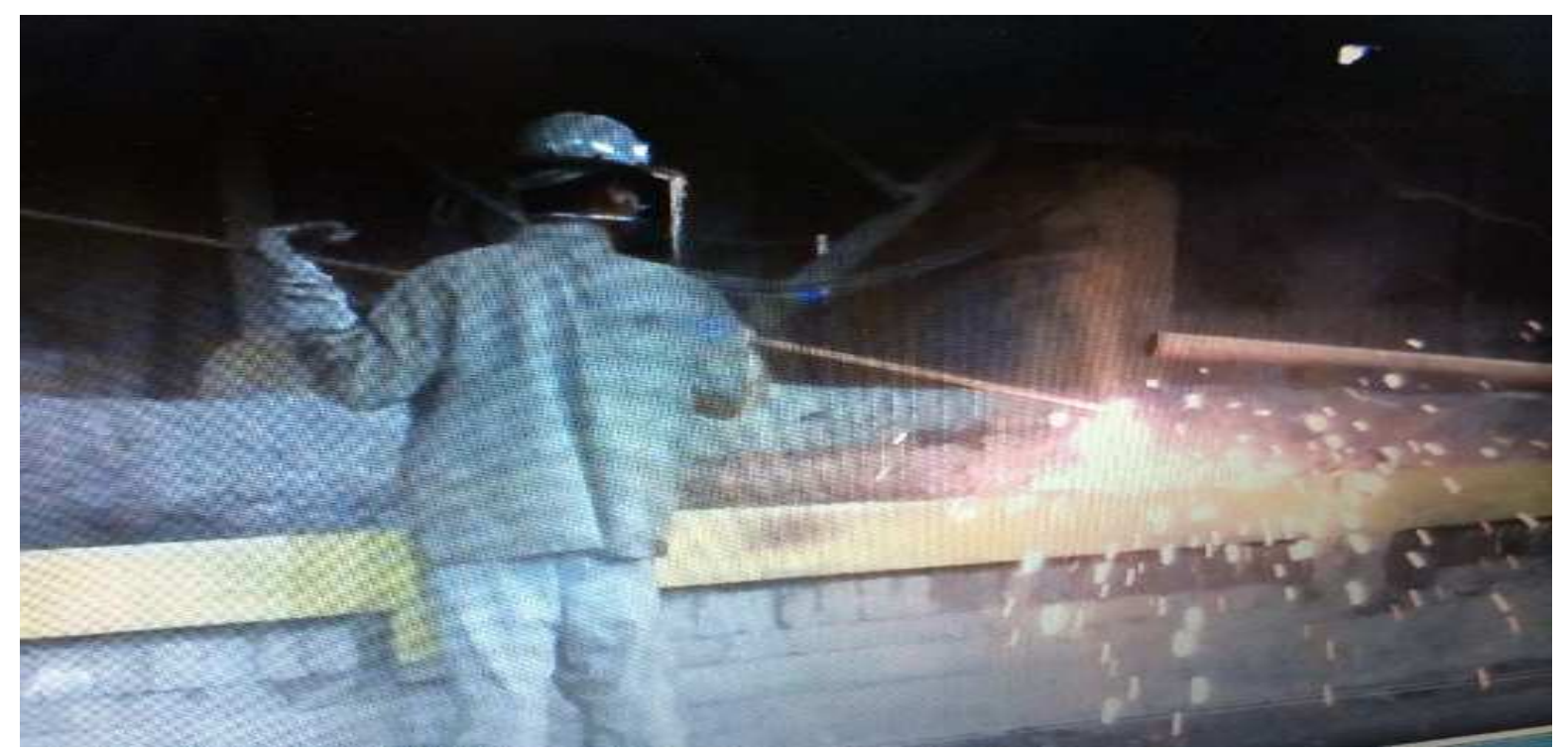

Figura 2 - processo manual de limpeza 
Podendo em casos de aderência excessiva, impedir sua retirada do interior do convertedor e ou levando a incidentes e interrupção da produção (4), como ilustrado na figura 3.

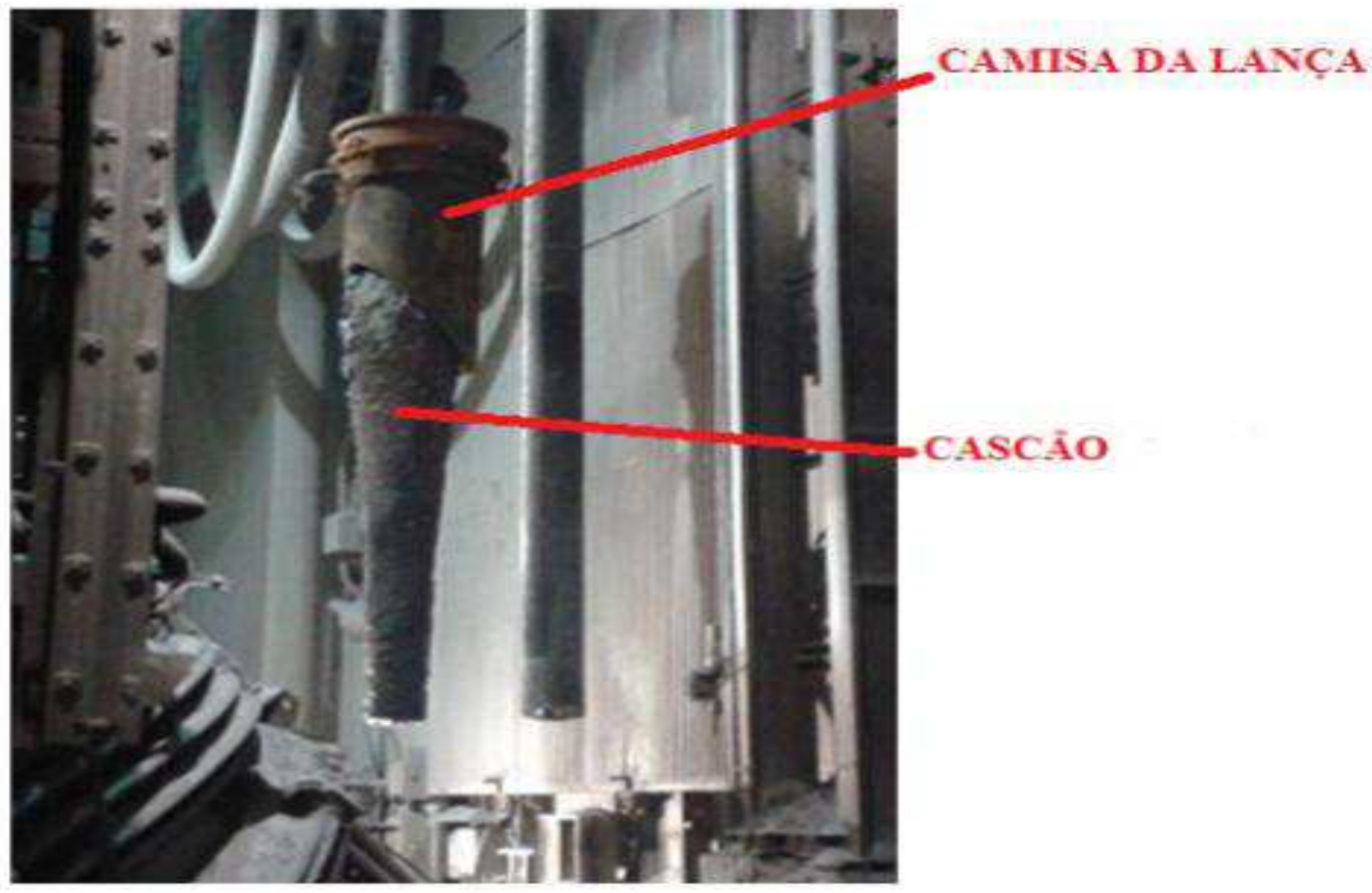

Figura 3 - Cascão excessivo - rompimento da camisa.

Para solucionar estes problemas de modo seguro e com alta performance é que se desenvolveu o dispositivo de remoção contínua de cascão incandescente aderido na superfície das lanças dos processos aplicados nos convertedores LD.

\section{MATERIAIS E MÉTODOS}

O dispositivo ( $R C L$ - MVL - 01), é formado por um suporte metálico simétrico bi partido, acionados por dois cilindros pneumáticos e controlados automaticamente por meio de sensores indutivos de posição aberto / fechado e ao PLC do carro de acionamento das lanças de injeção de oxigênio e gases inertes aplicadas nos convertedores LD, tendo sobre este suporte seis ferramentas de remoção do cascão, simetricamente distribuídas e confeccionadas em aços especiais de alta resistência e durabilidade (dupla face), três em cada lado do suporte, atuando sobre o cascão incandescente em mais de $95 \%$ do perímetro da lança e acopladas individualmente a cilindros mecânicos articulados via molas de compressão e mancais de modo a permitir sua variação / liberação na remoção / corte do cascão aderido sempre que superarem o limite da força de compressão aplicado as molas, como uma característica adicional de segurança (fig.4). 


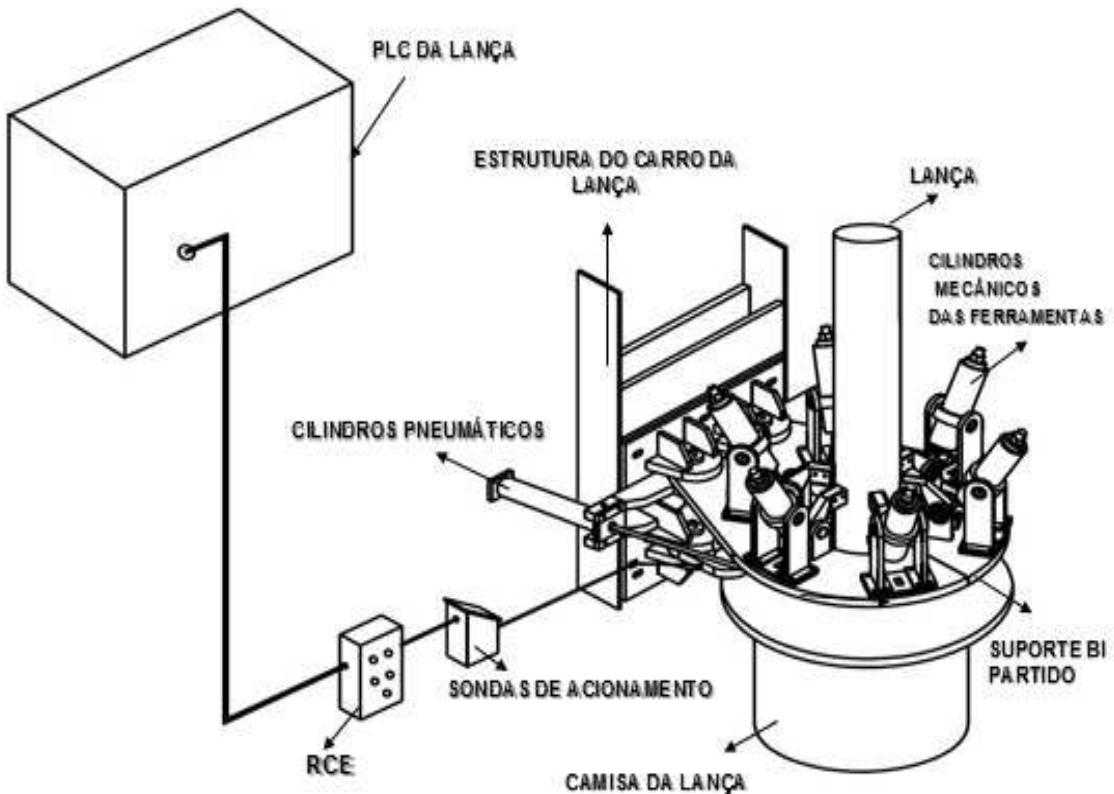

Figura 4 - disposição geral do dispositivo

Todo o conjunto de acionamento (Cilindros pneumáticos, cilindros mecânicos articulados via mancais / molas de compressão e sensores indutivos de posição), são revestidos com dupla camada de isolamento térmico (mantas) e chapas contra impactos mecânicos, (fig.5) além das chapas defletoras de proteção o que garante adequação ao meio de trabalho ou seja, altas temperaturas e impactos de materiais incandescentes.
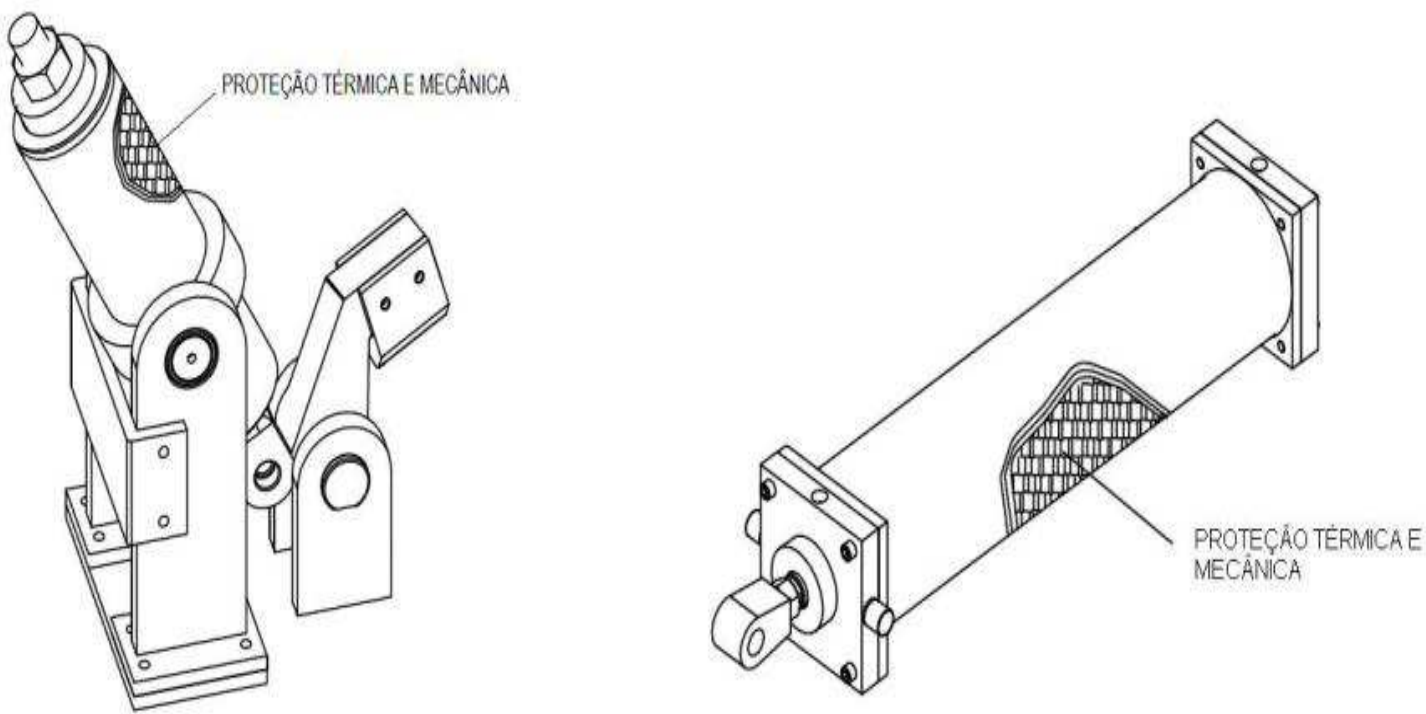

Figura 5 - llustração das proteções térmicas e mecânicas aplicadas no conjunto de acionamento do RCL - MVL - 01 .

Sua instalação e remoção foi desenvolvida empregando - se uma placa de base fixa (fig. 6), na qual se acopla por meio de parafusos uma placa de sustentação de todo o conjunto montado. Esta característica, tem a finalidade de facilitar a manutenção dos convertedores (reparos gerais) e do próprio dispositivo se necessário. Idealmente, o local de instalação do dispositivo (RCL) é acima da camisa da lança e na própria estrutura do carro vertical de movimentação das lanças (fig.7) 


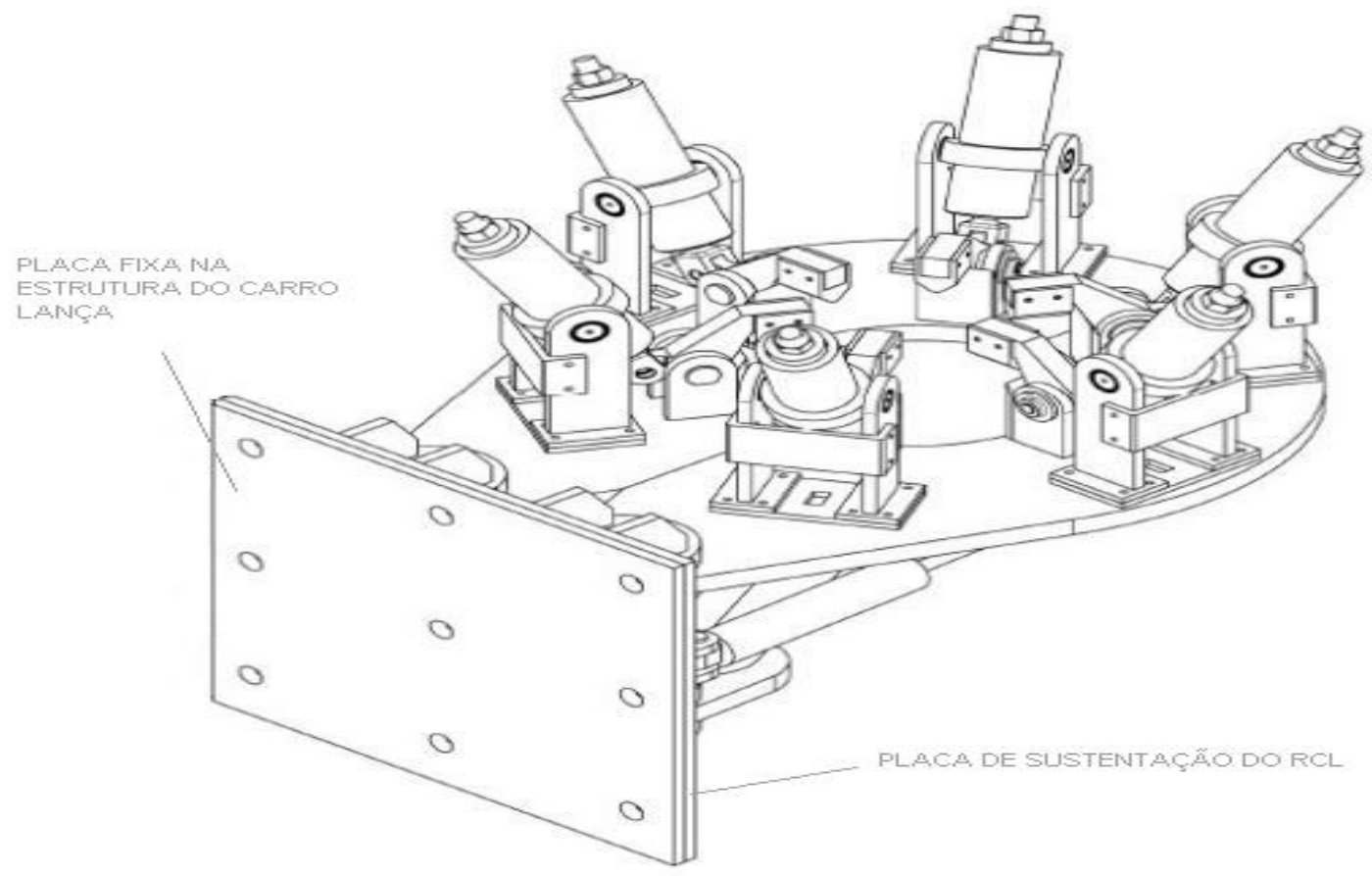

Figura 6 - fixação do conjunto RCL

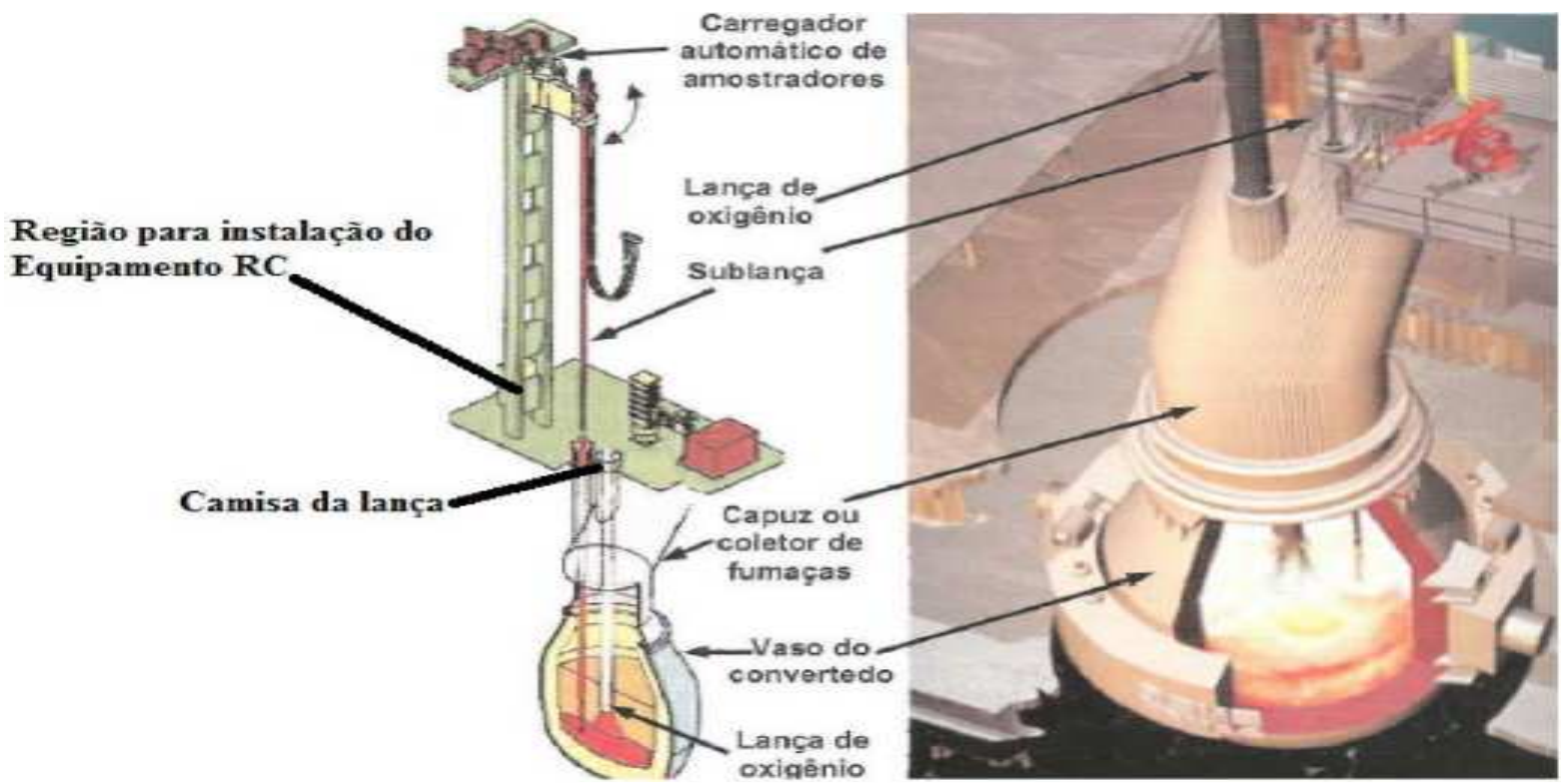

Figura 7 - llustração do posicionamento ideal RCL

Ressalta - se como diferencial a constante preocupação com a segurança operacional do dispositivo, sua durabilidade (garantia de alta performance) e facilidade de manutenção com baixa frequência e custo, bem como com todo o conjunto de movimentação das lanças, ao se empregar além das proteções necessárias ao severo ambiente de trabalho, controles automatizados através dos sensores de posição aberto e fechado (indutivos), circuito pneumático com válvulas reguladoras de pressão (fig.8), comandos elétricos / eletrônicos locais (RCE) fig.9, interligados ao PLC do carro de movimentação das lanças e os cilindros mecânicos com molas de compressão para articulação das ferramentas de remoção / corte do cascão. 


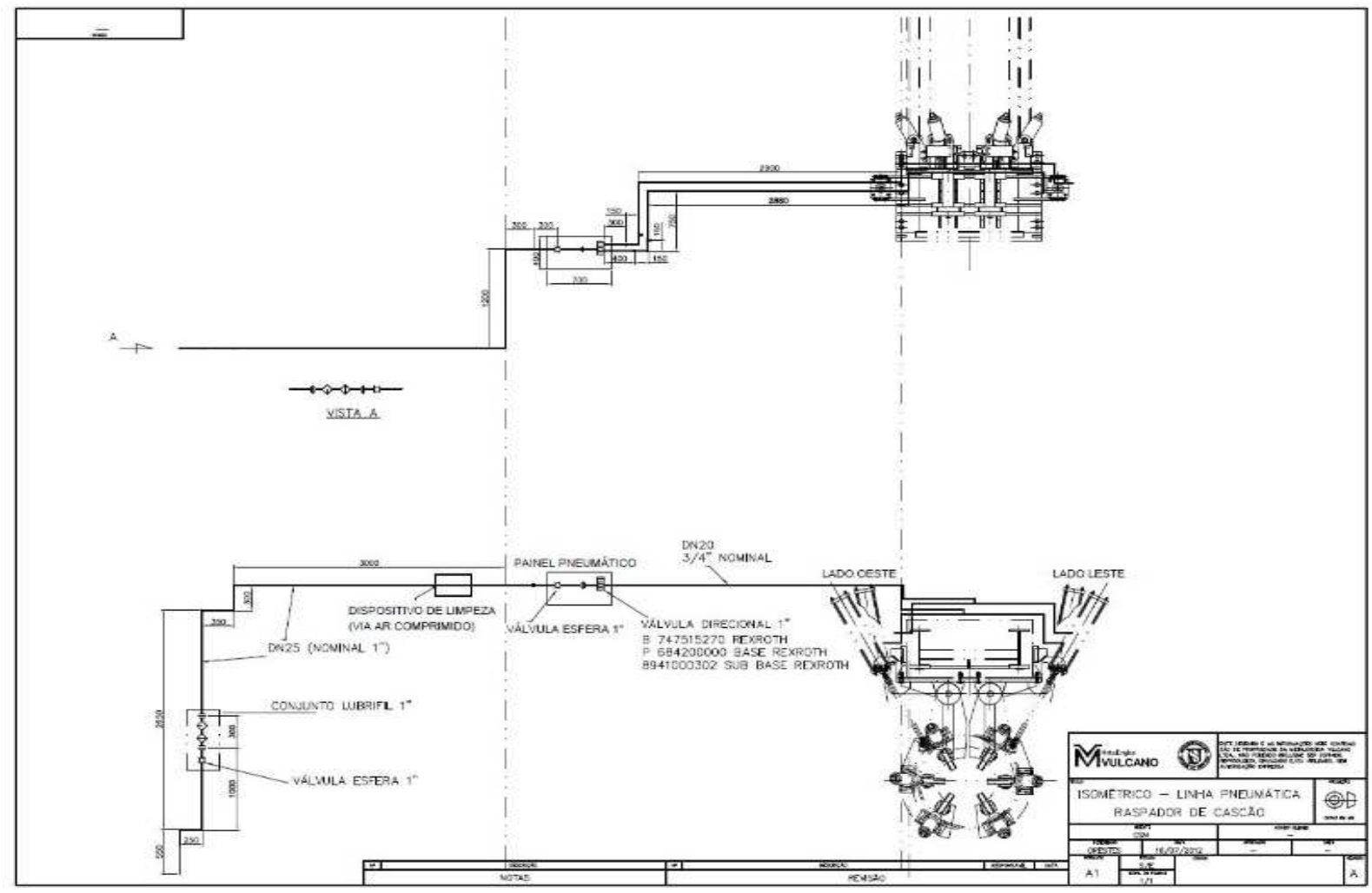

Figura 8 - Isométrico do sistema pneumático

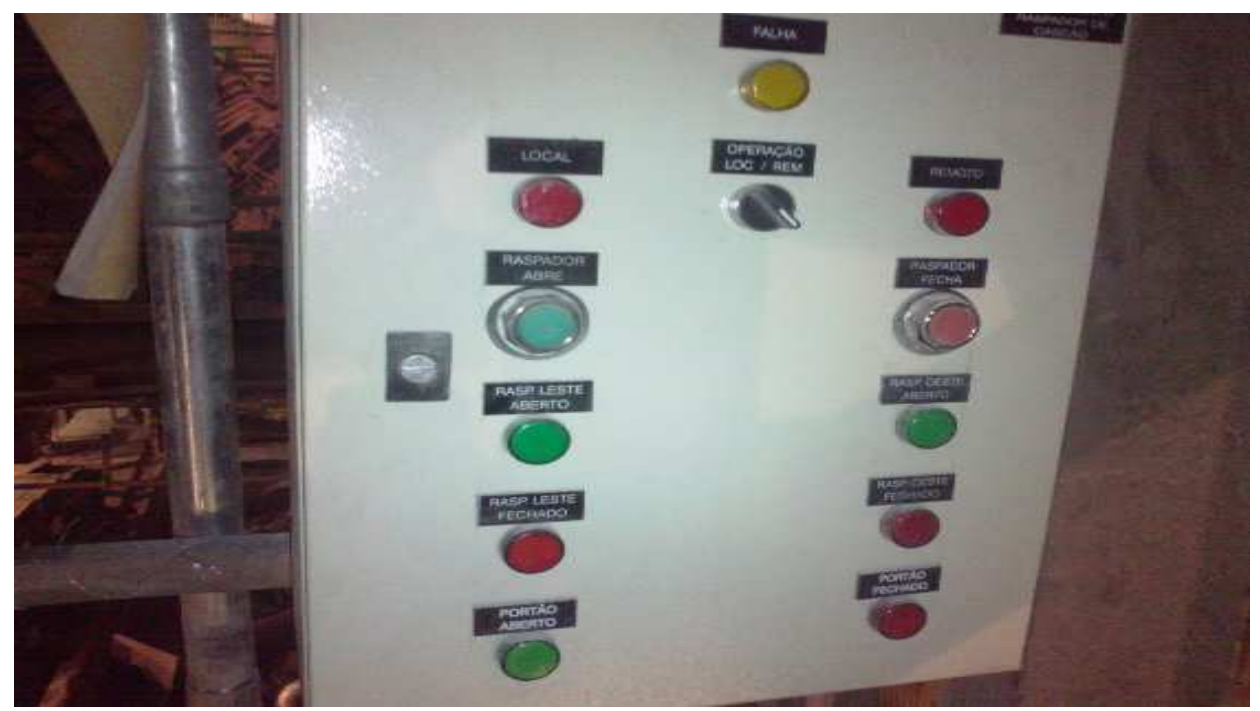

Figura 9 - Painel de controle local (RCE)

\section{RESULTADOS E DISCUSSÂO}

Com a aplicação industrial do presente dispositivo (RCL - MVL- 01), durante mais de 12 meses em um dos convertedores LD de uma planta siderúrgica, obteve - se sua aprovação com os seguintes resultados:

\subsection{Aumento da Vida Útil das Lanças}


Definindo - se vida útil das lanças como a quantidade de corridas sopradas sem seu afastamento por cascão aderido em sua superfície e com uma vida útil objetivada de 200 corridas (necessário para a troca da cabeça das lanças); Obteve se com uma taxa de utilização do dispositivo acima de $98 \%$, uma elevação média de $90 \%$ da vida útil média das lanças de 10 corridas para 190 corridas, correspondendo a $95 \%$ do valor objetivado; Conforme se apresenta na figura 10 de modo comparativo entre os convertedores A e B sem o dispositivo e o convertedor C com a aplicação do dispositivo

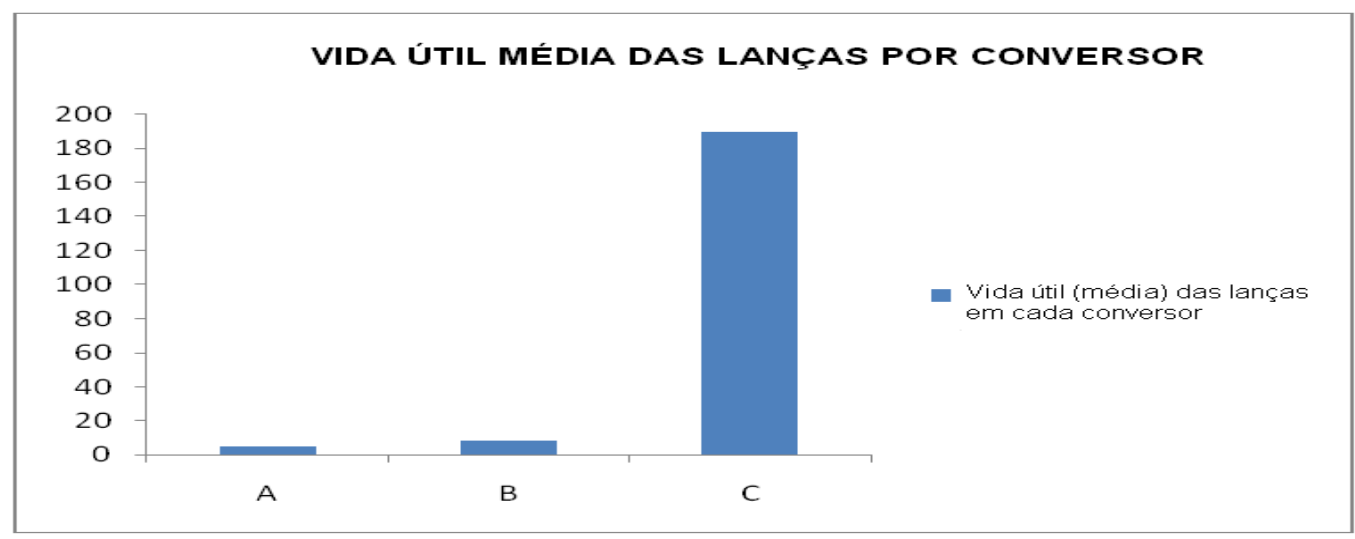

Figura 10. Vida útil das lanças com e sem aplicação do dispositivo RCL - MVL - 01.

Tal resultado, proporcionou expressiva estabilidade operacional dos processos de refino e "slag splash" no convertedor LD aplicado (convertedor C), com a eliminação da remoção do cascão via método manual (oxi - corte), resultando em aumento de produtividade com maior segurança e menor custo.

\subsection{Potencial de Ganho Econômico}

O quadro a seguir, apresenta o potencial de ganho econômico, avaliado no período de aplicação industrial do dispositivo ( $R C L$ - MVL - 01), considerando - se a redução de custo de limpeza do cascão das lanças por via manual (oxi - corte) e o potencial de ganho de produção em toneladas de aço líquido, pela maior disponibilidade do convertedor, deduzindo os dias de parada para reparos gerais (reformas) do convertedor LD. Observa - se que o valor da economia potencializado com a aplicação do dispositivo com uma reserva de dedução de $20 \%$, ou seja considerando $80 \%$ desse valor total; Possibilita uma taxa de retorno potencial de aproximadamente 4 meses no que se refere ao investimento a ser aplicado no presente dispositivo. 
Quadro de Potencial Econômico

\begin{tabular}{|c|c|}
\hline Custo de corte cascão por lança & 196,75 \\
\hline hh/lança & 8,33 \\
\hline $\mathrm{R} \$ \mathrm{hh}$ & 23,62 \\
\hline Lanças trocadas no período por cascão: & 877 \\
\hline$N^{\circ}$ de corridas entre trocas por cascão & 26 \\
\hline$N^{\circ}$ de corridas entre trocas por fim de vida & 241 \\
\hline$N^{\circ}$ de corridas por ano & 22800 \\
\hline$N^{\circ}$ dias no ano em que um dos conversores está em reforma & 40 \\
\hline$N^{\circ}$ de corridas nos períodos de reforma & 2499 \\
\hline$N^{\circ}$ de lanças trocadas por cascão no período de reforma & 96 \\
\hline Gasto com mão de obra no período: & $172.538,65$ \\
\hline Tempo de troca das lanças no período de reforma (minutos): & 1922 \\
\hline Tempo para troca de lança (minutos) & 20 \\
\hline $\begin{array}{l}\text { Potencial de aumento de produção no período (n॰ de } \\
\text { corridas) }\end{array}$ & 38 \\
\hline Tap to tap (minutos) & 50 \\
\hline Potencial de ganho total no período avaliado: & R\$ 4.097.386,87 \\
\hline Preço do aço líquido & USD 260,00 \\
\hline Câmbio & 1,7 \\
\hline Produção de aço por corrida (tonelada) & 231 \\
\hline Potencial de ganho de produção no período (tonelada) & 8880 \\
\hline Potencial de ganho financeiro no período & $\mathrm{R} \$ 3.924 .848,22$ \\
\hline Potencial de ganho financeiro total & $\mathrm{R} \$ 4.097 .386,87$ \\
\hline $\begin{array}{l}\text { Ganho: (período avaliado - } 12 \text { meses - considerando } 80 \% \\
\text { dos valores avaliados) }\end{array}$ & R\$ 3.277.909,49 \\
\hline
\end{tabular}

\section{CONCLUSÃO}

A aplicação industrial do (RCL - MVL - 01) dispositivo automatizado de remoção de cascão incandescente aderido na superfície das lanças de injeção de oxigênio e de gases inertes, utilizadas nos processos dos convertedores LD, possibilita afirmar a obtenção dos seguintes benefícios:

$\checkmark$ Maior disponibilidade dos convertedores LD, para atendimento ao programa de produção;

$\checkmark$ Menor consumo de lanças, pela sua maior durabilidade, possibilitando atingir sua vida útil objetivada ( $>=200$ corridas para troca por desgaste da cabeça / ponteira);

$\checkmark$ Maior segurança operacional, melhoria da qualidade do trabalho (saúde ocupacional - segundo as RN 12 e do meio ambiente RN 14), com a eliminação em mais de $95 \%$ das limpezas manuais das lanças (via oxi corte);

$\checkmark$ Flexibilidade de adequação do projeto básico aos ajustes dimensionais e operacionais de cada convertedor LD ("lay out”) e características do cascão formado;

$\checkmark$ Baixa frequência e facilidade de manutenção com baixo custo;

$\checkmark$ Taxa de retorno de alta viabilidade $(<=4$ meses - considerando o potencial de aumento de produção do aço líquido).

\section{Agradecimentos}

A Fundação de Amparo à Pesquisa do Estado do Rio de Janeiro FAPERJ Processo E-26/190.172/2009. Modalidade: ADT1. Que possibilitou 0 desenvolvimento e fabricação do RCL $-M V L-01$. 


\section{REFERÊNCIAS}

1 Ismar A.S. - Slagless lance recente results at BOF Steel Plants;

2 Malathi M, Brahma deo, boom, R - theorical and pratical aspect of lance skulling and slag foaming in BOF vessels;

3 Bramming Mats and Bjorkman, Bo - Avoiding sloppy process behavior AIStech 2010;

4 Nogueira A. Daniele, Formação e Remoção de cascão de lanças de oxigênio em conversores LD - Projeto de conclusão de curso de graduação e Engenharia Metalúrgica - UFF - EEIM - VR - Outubro- 2014. 